\title{
Percepção da síndrome de dependência por pacientes em tratamento
}

\author{
Dependence syndrome perception by patients undergoing treatment
}

Bruno José Barcellos Fontanella', Guilherme Arantes Mello', Marcelo Marcos Piva Demarzo', Egberto Ribeiro Turato²

\section{RESUMO}

A percepção da dependência de substâncias psicoativas é um dos principais motivadores da procura de tratamento por pessoas com este quadro clínico e a compreensão de seus significados constitui uma questão relevante em saúde. Objetivos: Levantar e compreender os significados pessoais atribuídos pelos pacientes que procuraram tratamento à síndrome de dependência. Método: Pesquisa qualitativa com entrevistas semidirigidas com amostra intencional de 13 dependentes de substâncias que procuraram tratamento. Resultados: Os atuais critérios para dependência de substâncias foram abordados espontaneamente pelos pacientes. Atribuíram variados significados pessoais à questão do desejo intenso, da compulsão e do descontrole quanto ao uso, e também à síndrome de abstinência, à tolerância, ao abandono de outros interesses e prazeres e à percepção das conseqüências nocivas do uso. Conclusão: Os entrevistados mostram compreensão própria do processo de procura de tratamento, avaliando meticulosa e subjetivamente o desconforto dos sintomas de dependência. Tais significados requerem especial atenção dos profissionais de saúde, sobretudo, nos atendimentos em atenção básica e, uma vez abordados, poderão facilitar a procura de tratamento.

\section{ABSTRACT}

The perception of the dependence symptoms is a key factor on the treatment seeking process by people who suffer from it, and its understanding is a relevant health issue. Objectives: The aim of the present study is to identify and understand the subjective meanings of the perceived dependence symptoms among patients who have sought treatment. Methods: Qualitative study conducted on an intentional sample of 13 substance dependents seeking formal treatment; in-depth semistructured interviews. Results: The diagnostic criteria on substance dependencewere spontaneously reported by the participants. They show a clear understanding of the process of treatment, compulsion and lack in controlling substance-taking behavior; withdrawal state; tolerance; neglect of alternative pleasures or interests; perception of harmful consequences due to substance abuse.

\section{Keywords}

Substance-related disorders, alcohol-related disorders, qualitative research.

Conclusions: The interviewees have a proper understanding on the seeking treatment process and seem to evaluate subjectively and carefully the distress related to the dependence symptoms. These results need special attention of health professionals, especially in primary care settings and, once approached, it may collaborate with treatment searching.

1 Departamento de Medicina do Centro de Ciências Biológicas e da Saúde da Universidade Federal de São Carlos (UFSCar), São Carlos, SP. 2 Departamento de Psicologia Médica e Psiquiatria da Faculdade de Ciências Médicas da Universidade Estadual de Campinas (UNICAMP), Campinas, SP.

$10 / 7 / 2008$ 
Os dependentes de substâncias psicoativas costumam identificar um conjunto de sintomas antes de um primeiro contato com os serviços de saúde ${ }^{1-4}$. Esta identificação é apenas potencialmente determinante da procura de tratamento, pois muitos deles não a efetivam ${ }^{5-7}$.

A percepção da gravidade do problema do uso de substâncias está associada positivamente à procura de ajuda e é também variável interveniente para outras variáveis de naturezas interpessoal e ambiental que atuam neste mesmo sentido ${ }^{8,9}$. Vários dos motivos alegados pelos usuários de substâncias que compuseram as amostras estudadas por Oppenheimer et al. e por Brooke, Fudala e Johnson², correlacionam-se a essa percepção da gravidade. No mesmo sentido, Bardsley e Beckman ${ }^{10}$ encontraram que um dos dois únicos elementos do modelo de crenças em saúde ${ }^{11}$ associados positivamente com a procura de tratamento por alcoolistas foi exatamente a percepção da gravidade; o outro elemento relaciona-se à percepção dos desencadeantes circunstanciais da procura. Na terminologia do modelo transteórico ${ }^{12,13}$, esta percepção contribuiria para a passagem do estágio de pré-contemplação para contemplação ${ }^{9}$ e, daí, para o estágio de ação.

Outras pesquisas ratificaram a importância da percepção da gravidade como disparadora da procura de tratamento, entre variados outros fatores associados ou facilitadores deste evento ${ }^{5,14-20}$. Oppenheimer et al. ${ }^{1}$ resumiram o processo de busca de tratamento por dependentes de substâncias psicoativas: ela resultaria da "combinação de eventos disparadores e uma reavaliação subjetiva do significado destes eventos".

De fato, o conjunto de fatores já estudados na literatura sugere que se procure aprofundamento destes significados (para o dependente), mais do que simplesmente a ampliação da lista das possíveis motivações envolvidas.

O entendimento desses significados adquire maior importância em um momento histórico brasileiro de valorização e reorganização do primeiro nível de atenção à saúde, traduzido pela implementação da estratégia de saúde da família que vem ocorrendo na grande maioria dos municípios $^{21}$. Tal estratégia proporciona aos médicos e às equipes de saúde envolvidas maior aproximação com as comunidades, e, conseqüentemente, com a realidade dos usuários de risco e dos dependentes de substâncias psicoativas, podendo, então, interferir positivamente na história natural dessa condição.

\section{Pressupostos teóricos utilizados}

A percepção de se ter um problema com alguma substância psicoativa pressupõe que ocorra com o usuário mudança no modo de avaliar alguns fenômenos até então considerados como egossintônicos. Tornam-se parcialmente egodistônicos, e o indivíduo percebe que há algo "errado" consigo. Tal mudança não altera, necessariamente, o prognóstico da situação - pois é freqüente desejar e não conseguir mudanças em um padrão alterado de uso de substâncias. Pode, porém, abrir um campo para mudanças espontâneas de comportamento e/ou intervenções clínicas.

Pode-se considerar que o desejo de vivenciar mudanças "para melhor" exista sempre, em algum nível, consciente ou não. Por outro lado, estaria também em jogo o desejo de usufruir as conseqüências biopsicossociais consideradas positivas do uso de substâncias, ou mesmo do estado de dependência. Haveria um momento em que esta situação ambivalente se desequilibraria, para alguns usuários, em favor da procura por tratamento. Compreender como vivenciam e percebem este movimento pode contribuir para melhor adequação dos serviços e dos profissionais às necessidades de saúde destas pessoas.

O benefício de recorrer a diferentes teorias do pensamento psicológico para a compreensão dos processos de mudanças de comportamento foi reconhecido por Prochaska e DiClemente ${ }^{12}$ na elaboração de seu "modelo transteórico de mudanças de comportamento", que tem exercido marcada influência sobre as pesquisas dessa área nas últimas décadas.

A teoria comportamental da motivação prevê que a partir da necessidade percebida como de autopreservação, que emerge depois de longa exposição às substâncias de que abusa, o dependente se dirija a uma das possíveis soluções para este estado, por exemplo, o impulso de busca de tratamento. Encontrá-lo funcionaria como incentivo positivo. Como geralmente ocorre neste processo, a pessoa pode também impulsionar-se para novos episódios de uso de substâncias, e avaliar se o incentivo do tratamento realmente satisfaz. Há ainda a influência de incentivos negativos, como a possibilidade de ineficácia terapêutica, o medo das técnicas utilizadas e os custos do tratamento.

De maneira complementar, a teoria cognitiva supõe que as representações conscientes das referidas necessidades gerem intenções e promovam planos para que se atinjam os objetivos: os indivíduos se motivariam por suas cognições a respeito dos aspectos que Ihes afligem na situação de dependência.

Já a teoria psicanalítica supõe que as motivações não podem ser inferidas somente a partir das ações ou cognições. Postula-se que muitos dos comportamentos humanos se relacionam a motivos dos quais os próprios sujeitos não têm consciência, reprimidos na esfera do inconsciente. Os fenômenos relacionados ao uso abusivo ou à dependência e seus sintomas atenderiam a necessidades inconscientes - benefícios primários da doença. A procura de ajuda não é vista como imediatamente vinculada à necessidade de solução pelo tratamento, mas como resultante de um conjunto complexo de investimentos pulsionais 
conflitivos, geralmente manifestados de maneira deformada, disfarçada e simbólica, como as outras manifestações do inconsciente.

Estas visões teóricas possuem elementos de semelhança, já que a teoria psicanalítica das pulsões (fonte, alvo e objeto da pulsão) apresenta paralelismo com a teoria comportamental-cognitiva da motivação (necessidade, impulso-intenção e incentivo).

Sumariamente expostas, elas são o pano de fundo para a análise feita nesta pesquisa. Parafraseando Thom ${ }^{14}$, os pressupostos sobre o processo de procura de tratamento por dependentes são assim resumidos: estariam em jogo numerosas contradições comportamentais e cognitivas, pressões e contrapressões sobre o indivíduo, algumas inconscientes, falsos começos e passos rastreados várias vezes. Esta evolução lenta da consciência da necessidade de ajuda fugiria, portanto, do processo puramente racional.

O objetivo específico deste trabalho foi compreender, a partir da visão de alguns pacientes, como a percepção das diretrizes ou dos critérios diagnósticos, do que a medicina denomina de síndrome de dependência, pode constituir-se em motivação para procura de tratamento formal.

\section{MÉTODO}

Foram feitas e analisadas entrevistas semidirigidas com questões abertas com pacientes dependentes de substâncias psicoativas que procuraram tratamento. Os princípios metodológicos qualitativos utilizados, descritos a seguir, foram sistematizados por um dos autores para aplicação específica nos settings de cuidado à saúde ${ }^{22}$.

\section{Amostragem}

Todos os participantes foram recrutados de dois serviços assistenciais onde eram pacientes do entrevistador (primeiro autor): um consultório particular e uma clínica de internação psiquiátrica da cidade de Campinas, SP.

A amostra foi constituída por 13 sujeitos com expressividade verbal suficiente para sustentar a entrevista em profundidade. Seis deles já haviam se submetido a tratamento prévio e os demais procuravam-no pela primeira vez. Foram entrevistados seguidamente nove sujeitos adequados aos critérios de inclusão, adiante explicitados, até que novas entrevistas não acrescentassem dados substancialmente novos àqueles já obtidos, segundo a crítica dos pesquisadores. Este fechamento amostral por saturação ocorreu conforme o descrito por Fontanella, Ricas e Turato ${ }^{23}$. Em seguida, foram incluídos quatro outros componentes por variedade de tipos (elementos que, por sua tipicidade quanto a certas características clínicas e sociodemográficas, preenchiam algumas lacunas da amostra heterogênea procurada pelos autores: uma mulher, um dependente de opiáceos, um dependente de crack-cocaína e um que chegou sob coerção da empresa onde trabalhava).

Os critérios de inclusão foram: aderir ao tratamento inicial (comparecimento por, pelo menos, três vezes ao consultório ou sete dias de internação); estar nas três semanas iniciais de tratamento; manter a adesão depois de resolvidas as situações agudas desencadeadoras da internação ou consulta (quadro agudo de síndrome de abstinência, comportamentos disruptivos com crises familiares, intoxicação aguda grave etc.); diagnóstico de dependência de, ao menos, uma substância psicoativa (CID-10: F1x.2); queixa principal relacionada a esse diagnóstico; ter ciência do diagnóstico; e concordar em participar da pesquisa.

\section{Coleta de dados: entrevistas}

O tema geral das entrevistas semidirigidas foi "como ocorreu sua procura de tratamento" e, secundariamente, abordaram-se os subtemas "fatores desencadeantes"; "o que entende por tratamento"; "aspectos do uso que deseja modificar"; "por que não procurou tratamento antes"; "como imaginou os profissionais que o atenderiam"; "como eles deveriam ser"; e "influência de tratamentos anteriores".

Procurou-se catalisar as reflexões dos participantes, que foram instados a exercer papel ativo na entrevista e se expressarem com suas próprias palavras, procurando o entrevistador (primeiro autor) modular as entrevistas segundo a estrutura psicológica particular de cada participante ${ }^{24}$. Outros procedimentos objetivaram maximizar a validade e a confiabilidade das entrevistas ${ }^{25}$ : estabelecer bom rapport entrevistador-entrevistado, considerar o entrevistador "como instrumento"24, manter um setting das entrevistas estável, sem grandes variações entre um entrevistado e outro e, finalmente, propor o mesmo tema geral e subtemas a todos os participantes.

\section{Análise dos dados}

As entrevistas foram transcritas por um técnico e revisadas pelo entrevistador. Sobre este corpus, realizou-se análise de conteúdo ${ }^{26}$ : os autores fizeram leituras das transcrições para se aprofundarem no material e formularem categorias descritivas, codificando as motivações e as barreiras à procura de tratamento relatadas pelos entrevistados e as induzidas pelos pesquisadores (processo decorrente da identificação de fenômenos clínicos, psicológicos e socioculturais nos comportamentos dos participantes). Procurou-se, também, maximizar a validade e a confiabilidade desta análise por meio de triangulação entre os analistas dos dados (os autores que, depois de codificações e análises independentes, discutiram as categorias consensuais e as que se tornaram consensuais), exemplificação e uso de teorias na discussão dos resultados, transcrição integral das entrevistas disponibilizadas a pesquisadores independentes à pesquisa, membros dos laboratórios de pesquisas qualitativas a que 
os autores se vinculam e informações sobre o contexto clínico-social vivido pelos entrevistados e a procura ativa de falseamento das hipóteses levantadas.

Esta pesquisa foi aprovada pelos comitês de ética do Departamento de Psicologia Médica e Psiquiatria da Faculdade de Medicina da Universidade Estadual de Campinas (UNICAMP), e todos os indivíduos assinaram termo de consentimento e esclarecido antes de qualquer procedimento do estudo fosse iniciado. No processo de coleta, análise e divulgação dos dados e resultados, garantiu-se sigilo em relação a quaisquer dados que pudessem indicar a identidade civil dos participantes.

\section{RESULTADOS}

Este artigo integra um conjunto de textos que discute o mesmo corpus de entrevistas ${ }^{27-29}$

A Tabela 1 apresenta as principais características dos 13 participantes. Trata-se de amostra intencionalmente heterogênea quanto a variáveis sociodemográficas e clínicas, incluindo a substância de uso. O corpus foi constituído de 19 horas de gravações de áudio transcritas (duração média de 90 minutos por entrevista).

Tabela 1. Caracterização da amostra: algumas variáveis sociodemográficas e clínicas.

\footnotetext{
A 50 anos, branco, casado, publicitário, desempregado. Dependente de álcool há 15 anos, uso nocivo de cocaína intravenosa há 20 dias.

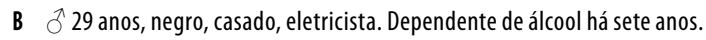

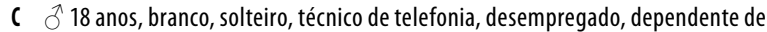
cocaína intranasal há dois anos.

D đ̋ 24 anos, branco, solteiro, sem profissão, não trabalha, dependente de cocaína intranasal há cinco anos.

E Ô 39 anos, branco, viúvo, engenheiro, empregado, dependente de álcool há dois anos.

F 36 anos, branco, casado, serralheiro, dependente de álcool há sete anos.

G 322 anos, branco, solteiro, comerciário, dependente de cocaína intranasal há três anos.

H 29 anos, branco, solteiro, comerciário autônomo, dependente de cocaína há dois anos, uso intranasal.

I 39 anos, branco, casado, desenhista, dependente de álcool há quatro anos.

J $q 20$ anos, parda, solteira, sem profissão, não trabalha, dependente de maconha há um ano.

K Ô 23 anos, pardo, solteiro, industriário, empregado, dependente de crack há um ano.

L đ 43 anos, pardo, casado, industriário, dependente de álcool há seis anos.

M 34 anos, branco, solteiro, aposentado por invalidez, dependente de codeína (por via oral, anteriormente por via intravenosa) há nove anos.
}

As categorias foram formuladas a partir da identificação e da seleção de temas valorizados pelos próprios entrevistados ou pelos autores, em vista dos referenciais teóricos utilizados.

Os entrevistados valorizaram, de maneira aparentemente espontânea, em suas reflexões sobre a procura de tratamento que efetivaram, os fenômenos que compõem a síndrome de dependência. Os achados foram organizados em seis categorias: desejo forte ou compulsão para usar e desejo de diminuir o uso; descontrole quanto ao início, ao término e aos níveis de consumo; percepção do estado de abstinência fisiológica; percepção da tolerância; abandono de interesses e prazeres alternativos; e percepção das conseqüências nocivas. Correspondem aproximadamente à lista de fenômenos que constituem a síndrome, de acordo com as diretrizes das duas principais classificações diagnósticas psiquiátricas atuais, CID-10 E DSM-IV30,31. São critérios cujas validades de conteúdo e construção vêm sendo verificadas desde a descrição preliminar da síndrome por Edwards e Gross ${ }^{32}$.

\section{Desejo forte ou compulsão para usar e desejo de diminuir o uso}

Vivenciar a compulsão foi a mais angustiante das percepções relatadas. Alguns entrevistados se sentiram irremediavelmente ligados ao uso continuado, perplexos diante da primazia que o uso da substância passou a ter em suas vidas:

"[...] é automático. Penso 'vou parar com isso, vou parar'. Aí, beleza... você fica um, dois, três dias... chega no terceiro ou quarto dia, você fica meio... Tendo dinheiro na mão, você usa. Sei lá, o negócio é meio esquisito." (C)

"Ela começa a gerar uma coisa assim... que você só vai pensar nela, entendeu? Você não pensa em mais nada. Só pensa nela mesmo." (G)

O relato de uma participante que não procurou tratamento espontaneamente (foi pressionada pela família) associou-se a uma fala emocionalmente indiferente sobre a compulsão, porém reveladora de que também a percebia:

"[...] acho que sou dependente, sim. Eu gosto, eu pre..., não chego a precisar. Já cheguei num ponto de ficar 'preciso, preciso, preciso'. [...] dou uns dois ou três tiros, eu não quero parar mais, também." (J)

Desejo intenso, compulsivo, fissura, foram mencionados pelo entrevistado $D$, que mostrou suas dificuldades emocionais de descrever este fenômeno, aparentemente racionalizando suas angústias diante da compulsão:

"... Eu acho que a fissura... essa tal vontade que a gente sente... é uma vontade que não existe... a gente sabe que não existe e passa... Aliás, ela não existe, a gente não vê, mas tá sentindo, tá precisando... Essa... de corpo pedir cocaína..., olha... não sei se meu corpo chegou a pedir cocaína, acho que mais minha cabeça, entendeu?" (D)

Outros exemplos mostram a intensidade e a singularidade do conflito entre a "vontade louca" (A), por um lado, e o desejo de não sucumbir ao uso, por outro lado:

"Procurei parar algumas vezes mas, face à vontade, à fissura que eles falam, é uma coisa que realmente era muito difícil [...] Como te explico? [...] fico pensando naquilo, me irrita, dá uma ansiedade." (H)

"... uma tremenda vontade... [...] uma vontade louca." (A)

"... pensava, pensava, voltava, mandava e bebia." (E)

Considerando os exemplos citados em seus respectivos contextos, inferiu-se que a compulsão, de certa forma, 
resume a relação de sofrimento dos entrevistados com as substâncias, da qual derivariam outras conseqüências consideradas negativas do uso. Não pareceram se preocupar ou teorizar com os mecanismos envolvidos nas origens dessa vivência, apenas expressaram se sentir à mercê deste automatismo volitivo, como referiu $\mathrm{K}$ :

"O crack é três segundos e você tá louco. Depois de quatro segundos você quer mais." (K)

A perplexidade diante de algo assim vivenciado pode estar subjacente à crença de que seus problemas não são compreendidos pelos clínicos, considerados incapazes de entender o que se passaria com seus pacientes, dificultando a procura por tratamento ${ }^{27}$. Como seria esperado pelas propriedades farmacológicas, tal perplexidade diante da compulsão ocorreu de maneira mais intensa nos entrevistados dependentes de cocaína (C, D, G, J e K) e codeína (M).

\section{Descontrole quanto ao início, ao término e aos níveis de consumo}

O uso que ocupa muito tempo foi fator mencionado espontaneamente pelos participantes. Queixaram-se do tempo gasto e da recuperação demorada da intoxicação, tirando o tempo de convívio com pessoas próximas. As seguintes frases se referem à sensação de descontrole quanto ao início de uma sessão de uso da substância:

"... é terrivel, porque antes de usar, você quer; depois que usou, se arrepende. Eera todos os dias. Na hora em que estava passando o efeito, usava de novo. Terrivel." (M)

"Às vezes eu ficava um dia sem usar. 'Eu vou parar.' Aí eu usava. Conforme usava, depois que passava o efeito, falava '[...] para que eu fiz isso?'." (K)

Estes exemplos se referem também ao arrependimento de não terem conseguido se controlar. Passaram, portanto, a se sentir culpados e incitados a rever seus padrões de uso de codeína e crack-cocaína. Isso parece ter ocorrido quando começaram a perceber a ineficácia de alguns mecanismos que vinham utilizando para manter a sensação de controle do uso: tentativas de interromper o caráter compulsivo do uso por meio do "descanso" (abstinência durante alguns dias da semana) e da "cotas de uso" (limites que não deveriam ultrapassar). Tais mecanismos constituiriam organização psicológica defensiva diante da angústia da vivência da compulsão, podendo ser classificadas como técnicas espontâneas de redução de danos que, até então, eram percebidas como eficazes ${ }^{28}$.

\section{Percepção do estado de abstinência fisiológica}

A percepção da síndrome de abstinência constituiu também fator gerador de angústia e desconforto. Entretanto, os participantes, em geral, não a enfatizaram como fator motivador da procura de tratamento, talvez por sentirem que aprenderam, segundo seus relatos, a lidar eficazmente com a síndrome em seus cotidianos. Este aprendizado pode ter servido como barreira para procura de tratamento mais precoce, à medida que era habilidade exercida sem ajuda externa.

A situação de $M$ é peculiar neste sentido, porque, não podendo mais injetar codeína, passou a administrá-la por via oral, sem obter os efeitos desejados e com tentativas de interrupção fortemente marcadas pela abstinência:

"Cada vez que a gente tenta parar... sozinho... O dia passa, o sofrimento... depois volta a tomar, é uma derrota, né? Fica pensando que não..., não tem jeito." (M)

\section{Percepção da tolerância}

Os significados dados pelos participantes à tolerância farmacológica foram variáveis. Para $\mathrm{D}$, o aumento das quantidades usadas foi visto como positivo durante algum tempo, conotando maior resistência física:

"Quinze chopes [...] Eu nem ficava mais bêbado, eu não ficava bêbado [...]." (D)

O participante M percebeu a tolerância como claro obstáculo à obtenção do efeito desejado porque a via de administração atual (oral, pois já não dispunha de veias acessíveis) não permitia concentração de codeína suficiente para obter tal efeito. Ele, então, se hospitaliza, "descansa", isto é, tenta diminuir a tolerância farmacológica, para depois da alta tentar reaver a possibilidade de prazer intenso com a substância:

"[...] depois que eu saí do hospital, voltei a usar drogas [...] meu corpo já tinha desacostumado. Então foi gradual, como no início, não foi tão freqüente." (M)

O participante $\mathrm{H}$ valorizou a tolerância como indício, mensurável objetivamente, que conotava problemas crescentes com a cocaína:

"No começo foi o embalo, mesma coisa de você tomar um porre no final de semana... Aí começou todo o dia, mas era gostoso de fazer. Todo mundo cheirava. Daí começam as depressões, e você sempre aumentando a quantidade. Tinha dia que cheguei a consumir quase 15 g." (H)

\section{Abandono de interesses e prazeres alternativos}

Os entrevistados se sentiram como "desperdiçando" outros lazeres, amizades e interesses. Perceberam-se alterando o ritmo cotidiano e mudando suas prioridades. As dificuldades com o trabalho foram particularmente relevantes para alguns:

"Você não tem vontade de trabalhar, de acordar cedo, de estudar. Ela prejudica em bastante coisa." (G)

"O trabalho... eu simplesmente..., praticamente abandonei, né? Eu não..., eu não sei se é... Atrasei a escola das crianças, ficou um monte de coisa pendurada... né?" (E)

Nos respectivos contextos, estas frases mencionadas anteriormente se referiam à perda da confiança de pessoas próximas, que também teriam percebido o abandono dos afazeres e dos interesses cotidianos dos entrevistados. 
Ocorre aqui, também, conotação de falha e culpa, explicitada na fala de C:

"Tem vez que você quer desistir de tudo. Quando parei, a segunda vez que parei [de ir à escola], comecei a faltar na escola pra cheirar, comecei a mentir." (C)

\section{Percepção das conseqüências nocivas}

As conseqüências físicas, relacionadas ao mau funcionamento do "corpo", foram valorizadas:

"Se você abusou muito, não come, seu corpo fica mais fraco, mais frágil, não continua aquilo. A coca, ela te dá mais ânimo do que você pode imaginar." (J)

"Vários fatores prejudiciais, tanto mental quanto no corpo; na mente, mental, os neurônios vão embora, você fica mais fraca, é mais desligado de tudo, você não tem vontade de fazer várias coisas... Mas é uma coisa que, temporariamente, está agradável..." (J)

O mesmo se observa em relação a conseqüências sociofamiliares:

"Antes vinha afetando meu lar, minha casa, meu casamento estava indo por água abaixo. Minha filha não podia me ver chegar e já passava mal. Então sabia que já estava prejudicando tudo." (L)

Várias conseqüências psicológicas, entre elas alguns déficits de funções cognitivas, foram valorizados:

"Seu raciocínio fica mais lento também, fica mais lento pra raciocinar. Tinha dias que eu não ia trabalhar, perdia a hora, não aparecia." (G)

"Você cheira cocaína até 4 horas da manhã, não dorme, fica até as 7 horas acordado. Aívocê não consegue trabalhar." (C)

\section{DISCUSSÃO}

O estudo de Edwards e Gross ${ }^{32}$ balizou o desenvolvimento dos critérios diagnósticos para dependência de álcool nas taxonomias propostas nas décadas seguintes, extensíveis hoje a outras substâncias. Tomaram como base, fundamentalmente, segundo os próprios autores, a "astuta observação da síndrome" pelos próprios pacientes. Observou-se no conjunto das entrevistas aqui trabalhadas que os pacientes de fato reconhecem plenamente os elementos do que a abordagem científica denomina síndrome de dependência, relacionando-os de maneira aparentemente "espontânea" com seus processos de procura de tratamento.

Presumiu-se, no entanto, a possibilidade de um processo de incorporação de tais critérios diagnósticos às representações sociais sobre a síndrome de dependência, dada a crescente divulgação pela mídia ou pelos profissionais de saúde dos elementos que a compõem, moldando o discurso desses entrevistados. Isso não invalidaria a associação entre reconhecimento da síndrome e procura de trata- mento, mas mudaria o modo de entender os fenômenos envolvidos. Os pacientes não estariam apenas validando a visão teórico-científica, mas sendo eles próprios influenciados pelo discurso científico.

Também é possível que os pacientes entrevistados tenham falado o que acreditaram que o profissional entrevistador queria ouvir ou poderia compreender, adaptando suas falas ao entrevistador. Esta possível restrição já havia sido apontada por Edwards e Gross ${ }^{32}$ e deve sempre ser considerada nos estudos qualitativos. $O$ fato de terem sido utilizadas entrevistas em profundidade, de durações relativamente longas, minimiza as possibilidades das influências do contexto em que elas ocorreram (pacientes diante de um médico que ao mesmo tempo era pesquisador) não tenham sido percebidas.

A pesquisa aparentemente se beneficiou desta abordagem metodológica, que possibilitou a expressão subjetiva de significados e lógicas pessoais. Embora a síndrome de dependência seja construto que corresponde ao conjunto estável de fenômenos (sinal disso é que tem mantido sua validade conceitual e de construção nas últimas três décadas), deve-se considerar que as constantes mudanças socioculturais no padrão populacional de uso de substâncias, assim como as histórias e problemáticas pessoais de cada entrevistado ou paciente, fornecem um pano de fundo menos estável e, dessa forma, sempre importantes de serem mais bem reconhecidas.

Independentemente das origens do discurso dos pacientes entrevistados, tem sido constatado epidemiologicamente que os fenômenos característicos da síndrome de dependência são fatores motivacionais presentes entre os que procuraram tratamento. Os usuários valem-se das noções de necessidade, freqüência de uso e descontrole, para avaliar suas condições ${ }^{19}$. Essas noções constituem fatores motivacionais de alto impacto e alta freqüência entre os que procuram tratamento!.

\section{CONCLUSÃO}

Conclui-se que os resultados são argumentos para indicar que os profissionais de saúde se capacitem a abordar os significados relacionados à busca de tratamento e, assim, contribuam para aumentar a reflexão consciente dos usuários de substâncias sobre a síndrome de dependência. Isso parece particularmente relevante nos serviços de atenção primária à saúde, porta de entrada preferencial para o sistema e um dos cenários onde as intervenções breves têm-se mostrado efetivas ${ }^{33}$. Investigar as associações feitas pelos pacientes talvez contribua para futura motivação para tratamento específico.

Considerou-se que os resultados descritos ajudam os clínicos a aperfeiçoar sua competência cultural com os 
pacientes usuários de substâncias, ou seja, os auxiliam a se inteirar de possíveis valores e lógicas pessoais, crenças e reações de seus pacientes.

Como costuma ocorrer nas pesquisas qualitativas, que não utilizam amostras probabilísticas, a validade externa dos resultados apresentados somente pode ser aferida pelos próprios leitores deste texto, caso os considerem plausíveis e aplicáveis aos pacientes e aos contextos específicos em que trabalham.

A inclusão de representantes de algumas subpopulações específicas de usuários e dependentes de substâncias poderia ter acrescentado outros elementos à discussão, como pacientes idosos, pois se sabe que neles a síndrome de abstinência se apresenta de maneira nem sempre típica; representantes de subpopulações menos expostas a influências culturais da mídia e do sistema de saúde, como residentes de zona rural, pessoas sem-teto etc., mulheres adultas e usuários que nunca procuraram tratamento, que poderiam em tese perceber a síndrome de dependência de maneira bastante diversa.

\section{AGRADECIMENTOS}

Baseado na tese de doutorado em Ciências Médicas do primeiro autor "Procura de tratamento por dependentes de substâncias psicoativas: um estudo clínico-qualitativo" (UNICAMP, 2000).

\section{REFERÊNCIAS}

1. Oppenheimer E, Sheehan M, Taylor C. Letting the client speak: drug misusers and the process of help seeking. Br J Addict. 1988;83:635-47.

2. Brooke D, Fudala PL, Johnson RE. Weighing up the pros and cons: help-seeking by drug misusers in Baltimore, USA. Drug Alcohol Depend. 1992;31(1):37-43.

3. Nielsen AS. Alcohol problems and treatment: the patients' perceptions. Eur Addict Res. 2003;9(1):29-38.

4. Tucker JA, Vuchinich RE, Rippens PD. A factor analytic study of influences on patterns of help-seeking among treated and untreated alcohol dependent persons. J Subst Abuse Treat. 2004;26(3):237-42.

5. Hingson R, Mangione T, Allan M, Scotch N. Seeking help for drinking problems: a study in the Boston Metropolitan Area. J Stud Alcohol. 1982;43:273-88.

6. Tucker JA, Galdsjo JA. Help-seeking and recovery by problem drinkers: characteristics of drinkers who attended Alcoholics Anonymous or formal treatment or who recovered without assistance. Addict Behav. 1993;18(5):529-42.

7. Wells JE, Horwood LJ, Fergusson DM. Reasons why young adults do or do not seek help for alcohol problems. Aust N Z J Psychiatry. 2007;41(12):1005-12.

8. Power R, Hartnoll R, Chalmers C. Help-seeking among illicit drug users: some differences between a treatment and nontreatment sample. Int J Addict. 1992;27(8):887-904.
9. Finney JW, Moss RH. Entering treatment for alcohol abuse: a stress and coping model. Addiction. 1995;90(9):1223-40

10. Bardsley PE, Beckman LJ. The health belief model and entry into alcoholism treatment. Int J Addict. 1988;23(1):19-28.

11. Cummings KM, Jette AM, Rosentock IM. Construct validation of the Health Belief Model. Health Educ Monographs. 1978;6(4):394-405.

12. Prochaska JD, DiClemente CC. Stages and processes of self-change of smoking: toward an integrative model of change. J Consult Clin Psychol. 1983;51(3):390-5.

13. DiClemente $\mathrm{CC}$, Hughes SO. Stages of change profiles in outpatient al coholism treatment. J Subst Abuse Treat. 1990;2(2):217-35.

14. Thom B. A process aproach to womens's use of alcohol services. Br J Addict. 1984;79(4):377-82.

15. Chitwodd DD, Morningstar $P C$. Factors which differentiate cocaine users in treatment from nontreatment users. Int J Addict. 1985;20(3):449-59.

16. Pfeiffer W, Feuerlein W, Brenk-Schulte E. The motivation of alcohol dependents to undergo treatment. Drug Alcohol Depend. 1991;29:87-95

17. Solís LR, Medina-Mora ME. La utilización de los servicios de atención para la salud mental por mujeres mexicanas: resultados de dos encuestas. Salud Mental. 1994;17(1):7-10.

18. Tucker JA. Predictors of help-seeking and the temporal relationship of help to recovery among treated and untreated recovered problem drinkers. Addiction. 1995;90(6):805-9.

19. Varney SM, Rohsenow DJ, Dey AN, Myers MG, Zwick WR, Monti PM. Factors associated with help seeking and perceived dependence among cocaine users. Am I Drug Alcohol Abuse. 1995;21(1):81-9.

20. Longshore $D$. Help-seeking by african american drug users: a prospective analysis. Addict Behav. 1999;24(5):683-6.

21. Brasil. Ministério da Saúde. Secretaria de Atenção à Saúde. Departamento de Atenção Básica. Política nacional de atenção básica. Braślia: Ministério da Saúde. 60 p. Normas e Manuais Técnicos. Série Pactos pela Saúde 2006, v. 4, 2006.

22. Turato ER. Tratado da metodologia da pesquisa clínico-qualitativa. Petrópolis, RJ: Vozes; 2003.

23. Fontanella BJB, Ricas J, Turato ER. Amostragem por saturação em pesquisas qualitativas em saúde: contribuições teóricas. Cad Saude Publica. 2008;24(1):17-27.

24. Bleger J. Temas de psicologia: entrevista e grupos. São Paulo: Martins Fontes; 1989

25. Fontanella BJB, Campos CJG, Turato ER. Data collection in clinical-qualitative research: use of non-directed interviews with open-ended questions by health professionals. Rev Latino-Americana Enfermagem. 2006;14(5):812-20.

26. Bardin L. Análise de conteúdo. Lisboa: Edições 70; 1979.

27. Fontanella BJB, Turato ER. Doctor-patient relationship barriers to substance dependents seeking treatment. Rev Saude Publica. 2002;36(4):439-447.

28. Fontanella BJB, Turato ER. Spontaneous harm reduction: a barrier for substance-dependent individuals seeking treatment? Rev Bras Psiquiatria. 2005;27(4):272-7.

29. Fontanella BJB, Turato ER. Percepção de sintomas depressivos por dependentes de substâncias psicoativas procurando tratamento. J Bras Psiquiatria. 2005;54(4):278-84.

30. OMS. Classificação de transtornos mentais e de comportamento da CID-10. Porto Alegre: Artes Médicas; 1993.

31. American Psychiatric Association. DSM-IV-TR-TM - Manual diagnóstico e estatístico de transtornos mentais. Porto Alegre: Artmed; 2002.

32. Edwards G, Gross MM. Alcohol dependence: provisional description of a clinical syndrome. BMJ. 1996;:1:1058-61.

33. Kaner EF, Beyer F, Dickinson HO, Pienaar E, Campbell F, Schlesinger C, et al. Effectiveness of brief alcohol interventions in primary care populations. Cochrane database of systematic reviews (0n-line). 2007;18(2):CD004148. 